\title{
Self-reported oral hygiene habits and periodontal symptoms among postpartum women
}

\author{
Alessandro Villa $\cdot$ Silvio Abati $\cdot$ Laura Strohmenger • \\ Marco Cargnel $\cdot$ Irene Cetin
}

Received: 14 December 2010/ Accepted: 19 April 2011

(C) Springer-Verlag 2011

\begin{abstract}
Purpose The purpose of this study is to describe oral hygiene practices and periodontal symptoms among postpartum women and to assess whether self-reported periodontal symptoms are correlated with a clinical diagnosis of periodontal disease.

Methods A self-administered questionnaire was developed to assess socio-demographic information, oral hygiene habits and frequency of dental visits among a group of 409 postpartum women. Additional questions included information on self-reported periodontal symptoms. Pregnancy information was obtained from medical charts.

Results $99.3 \%$ of the participants brushed their teeth every day; 46.2 and $53.8 \%$ reported teeth brushing once a day or more, respectively. Periodontal disease was present in $61.4 \%$ of women. Patients with gum swelling and tooth mobility were almost twice as likely to have a periodontal disease (OR 1.7, CI 95\% 0.9-2.8 and OR 1.7, CI 95\%: $0.8-3.5$, respectively). No associations were found between periodontal disease, gum swelling PTB and/or LBW.

Conclusions Maternal clinical periodontal disease at delivery was related with self-perceived gum swelling and tooth mobility. Dentists and prenatal medical care providers may have the opportunity to target pregnant women that
\end{abstract}

\footnotetext{
A. Villa $(\varangle) \cdot$ S. Abati - L. Strohmenger · M. Cargnel Dental Clinic, Department of Medicine, Surgery and Dentistry, University of Milano, Via Beldiletto 1/3, 20142 Milan, Italy e-mail: alessandro.villa@unimi.it

I. Cetin

Unit of Obstetrics and Gynecology, Department of Clinical

Science L. Sacco, University of Milan, Milan, Italy
}

might be at higher risk for periodontal disease by asking whether they have tooth mobility and/or swollen gums.

Keywords Oral hygiene habits - Symptoms · Periodontal disease $\cdot$ Pregnancy

\section{Introduction}

Oral diseases, such as gingivitis, periodontitis and dental caries, are prevalent conditions that affect individuals' oral health and may also have an impact on general health and quality of life [1]. Oral health during pregnancy can be characterized by frequent gingival changes [2]. Periodontal disease is a destructive inflammatory condition of the gum and bone that support teeth. Pregnant women may be more susceptible to periodontal disease since higher concentrations of progesterone, estrogen and synthesis of prostaglandins can induce edema, hyperemia, and bleeding in periodontal tissues [3], increasing the risk of bacterial infections. Periodontal pathogens, which can be reservoirs for pro-inflammatory mediators, may cross the placenta barrier and pose a potential threat to the fetus, thereby increasing the likelihood of an adverse pregnancy outcome [4]. Moreover, there is conclusive evidence that cariogenic bacteria in mothers can be transmitted to infants, even before tooth eruption, leading to increased dental caries risk in their children $[5,6]$. Therefore, maintaining a high standard of oral hygiene will contribute not only to the mother's general wellbeing, but will also positively affect the health of the newborn.

Understanding oral hygiene habits among pregnant women will help clinicians in planning interventions to improve both maternal and infant oral health. As such, the aim of this paper is to describe oral hygiene practices and 
periodontal symptoms among postpartum women and to assess whether self-reported periodontal symptoms are correlated with a clinical diagnosis of periodontal disease.

\section{Methods}

Study methodology has been previously described in detail [7]. Briefly, postpartum women were approached on the postnatal ward of the Hospital Policlinico, Mangiagalli and Regina Elena in Milan and were asked to complete a selfadministered questionnaire. All participants gave written informed consent. The study was approved by the Italian Ministry of University and Research and by the Scientific Committee of the Center for Fetal Research "Giorgio Pardi".

The questionnaire was developed to assess women's socio-demographic information, oral hygiene procedures, and frequency of dental visits. This questionnaire was similar to one that had been previously validated and employed in another study on pregnant women [8]. Oral hygiene habits, such as frequency of tooth brushing (brushing teeth once a day or more than once a day), time dedicated to tooth brushing ( $<1 \mathrm{~min}$, between 1 , and $3 \mathrm{~min}$ and more than $3 \mathrm{~min}$ ), and visits to a dentist (at least once a year, every 2 years, or when in pain) were assessed. Additional questions included information on self-reported halitosis, gum bleeding, gum swelling, and tooth mobility. All information was investigated by closed-ended question. Relevant data on pregnancy care and outcome were sourced from the medical records of the woman and her infant.

Finally, all women received a full-mouth dental and periodontal examination. Several measures of periodontal and dental health were then collected: gingival pocket depth, gingival recession, bleeding on probing (BOP), clinical attachment level (CAL), and decay missing filled tooth (DMFT). BOP was expressed as the percentage of sites exhibiting this response. For the purpose of this analysis, periodontal disease was defined as having CAL greater than or equal to $4 \mathrm{~mm}$ on 3 or more sites [9]. Patients were given the opportunity to receive periodontal therapy after dismissal from the clinic.

\section{Statistical analysis}

We evaluated the distribution of participant characteristics, including demographics, smoking status, and oral hygiene habits in Table 1; participants were given the option of refusing to answer each of the questions on the survey, and these refusals were treated as missing observations. In addition, data regarding type of delivery, newborn sex, and pregnancy outcome (preterm birth and low birth weight) were gathered. The threshold value for preterm birth (PTB) was set at $<37$ weeks of gestation and for low birthweight (LBW) at $<2,500 \mathrm{~g}$. Mean and standard deviations were calculated for CAL, BOP, DMFT, newborn weight and gestational weeks. Adverse pregnancy outcome was defined as having PTB and/or LBW.

We conducted a logistic regression analysis to evaluate, by means of odds ratios (ORs) and $95 \%$ confidence intervals (CI), relationships between periodontal disease, selfreported periodontal symptoms and adverse pregnancy outcome.

\section{Results}

A total of 409 women were included in this analysis (Table 1). The majority of study participants were European $(79.5 \%)$. Women ranged in age from 20 to 44 years, with a median age of 33 years. Nearly $61.3 \%$ of patients came from an urban area, 31.8\% from a suburban neighborhood, and $7 \%$ from a rural area. Around $32 \%$ of women completed University, $66.8 \%$ reported from 9 to 13 years of education, and $17 \%$ reported less than 8 years of education. Approximately $8.3 \%$ of the study sample reported current smoking.

When oral hygiene habits were considered (Table 1), $99.3 \%$ of the participants brushed their teeth every day; 46.2 and $53.8 \%$ reported teeth brushing once a day or more, respectively. Approximately $15 \%$ of women brushed their teeth for more than $3 \mathrm{~min}$ per day, $64.8 \%$ from 1 to $3 \mathrm{~min}$ and $21.5 \%$ for $<1 \mathrm{~min}$. Participants most frequently reported going to the dentist annually (59.9\%), although a relatively high proportion attended only when in pain (34.7\%); 5.4\% went every 2 years. Periodontal disease (3 or more sites $\geq 4 \mathrm{~mm} \mathrm{CAL}$ ) was present in 251 women (61.4\%); overall, the mean CAL was $2.2 \mathrm{~mm}$, the mean frequency of sites with BOP was $9.8 \%$ and the mean value of DMFT was 7.7.

The majority of women had a vaginal delivery (61.4\%). The incidence of deliveries at $\leq 37$ weeks' gestation (PTB) was $17.1 \%$ and LBW occurred in $13.4 \%$ of patients. The mean weight of newborns was $3,033 \mathrm{~g}$ and the mean gestation period was 38.3 weeks.

Table 2 shows associations between self-reported periodontal symptoms and clinical diagnosis of periodontal disease. Participants with gum swelling and tooth mobility were almost twice as likely to have periodontal disease (OR 1.7, CI 95\% 0.9-2.8 and OR 1.7, CI 95\% 0.8-3.5, respectively). No significant associations were observed for halitosis and gum bleeding.

Finally, when adverse pregnancy outcome was considered, no significant associations were found between PTB 
Table 1 Sociodemographic characteristics, oral hygiene habits and pregnancy outcome among 409 postpartum women

\begin{tabular}{|c|c|}
\hline & $\begin{array}{l}\text { Total }(N=409) \text {, } \\
n(\%)\end{array}$ \\
\hline \multicolumn{2}{|c|}{ Sociodemographic characteristics } \\
\hline \multicolumn{2}{|l|}{ Country } \\
\hline European countries & $325(79.5)$ \\
\hline Other & $84(20.5)$ \\
\hline \multicolumn{2}{|l|}{ Age category } \\
\hline $20-24$ & $23(5.6)$ \\
\hline $25-29$ & 59 (16.9) \\
\hline $30-34$ & $162(39.6)$ \\
\hline $35-39$ & $125(30.6)$ \\
\hline $40+$ & $30(7.3)$ \\
\hline Median [IQR] & $33[30-36]$ \\
\hline \multicolumn{2}{|l|}{ Education (years) } \\
\hline$<8$ & $7(1.7)$ \\
\hline $9-13$ & $273(66.8)$ \\
\hline 14 or more & $129(31.5)$ \\
\hline \multicolumn{2}{|l|}{ Area } \\
\hline Urban & $251(61.3)$ \\
\hline Sub-urban & $130(31.8)$ \\
\hline Rural & $28(6.9)$ \\
\hline \multicolumn{2}{|l|}{ Smoking status } \\
\hline Never & $255(62.4)$ \\
\hline Former & $120(29.3)$ \\
\hline Current & $34(8.3)$ \\
\hline \multicolumn{2}{|l|}{ Oral hygiene habits } \\
\hline \multicolumn{2}{|l|}{ Daily tooth brushing } \\
\hline Yes & $406(99.3)$ \\
\hline No & $3(0.7)$ \\
\hline \multicolumn{2}{|c|}{ Tooth brushing frequency } \\
\hline 1/day & $189(46.2)$ \\
\hline$+1 /$ day & $220(53.8)$ \\
\hline \multicolumn{2}{|c|}{ Daily time dedicated to teeth brushing (min) } \\
\hline$<1$ & $88(21.5)$ \\
\hline $1-3$ & $261(63.8)$ \\
\hline $3+$ & $60(14.7)$ \\
\hline \multicolumn{2}{|l|}{ Dental visit frequency } \\
\hline Annually & $245(59.9)$ \\
\hline Biennially & $22(5.4)$ \\
\hline Pain $^{\mathrm{a}}$ & $142(34.7)$ \\
\hline CAL (mm) & $2.2 \pm 0.5^{\mathrm{b}}$ \\
\hline Frequency BOP $(\%)$ & $9.8 \pm 14.6^{\mathrm{b}}$ \\
\hline DMFT & $7.7 \pm 4.2^{\mathrm{b}}$ \\
\hline \multicolumn{2}{|l|}{ Pregnancy outcome } \\
\hline \multicolumn{2}{|l|}{ Delivery } \\
\hline Vaginal & $251(61.4)$ \\
\hline Cesarean & $158(38.6)$ \\
\hline
\end{tabular}

Table 1 continued

\begin{tabular}{ll}
\hline & $\begin{array}{l}\text { Total }(N=409), \\
n(\%)\end{array}$ \\
\hline PTB & \\
No & $339(82.9)$ \\
Yes & $70(17.1)$ \\
LBW & \\
No & $354(86.6)$ \\
Yes & $55(13.4)$ \\
Newborn weight $(\mathrm{g})$ & $3033 \pm 650^{\mathrm{b}}$ \\
Gestational weeks & $38.3 \pm 2.5^{\mathrm{b}}$
\end{tabular}

$I Q R$ intra-quartile range, $C A L$ clinical attachment level, $B O P$ bleeding on probing, $D M F T$ decay missing filled tooth, $S D$ standard deviation, $P T B$ preterm birth was defined as a gestation of $<37$ weeks, $L B W$ low birth weight was defined as a weight of $<2,500 \mathrm{~g}$ (up to and including $2,499 \mathrm{~g})$

${ }^{a}$ Indicate patients visiting a dentist only when in pain

b Values given are mean \pm SD

and/or LBW and periodontal disease or gum swelling (Table 3).

\section{Discussion}

This study evaluated oral hygiene practices and the relationship between self-reported periodontal symptoms and periodontal disease among a large group of postpartum women. Previous analyses assessed the validity of selfreported measures for periodontal disease [10-12]; however, the results are controversial [13] and none of the studies to date focused on pregnant or postpartum women. We found that maternal clinical periodontal disease at delivery was related with self-perceived gum swelling and tooth mobility. Also, our findings show that only half of the women brushed their teeth more than once a day and the majority of participants dedicated 1-3 min to this procedure. In addition, a high proportion of patients (35\%) reported seeking oral care from a dentist only when they experience pain, thus making preventative strategies less possible. Indeed, periodontal disease was present in more than half of the women $(61 \%)$. Pregnancy gingivitis usually occurs from 30 to $100 \%$ of pregnant women [2]. Our study sample showed a relatively high proportion of women with periodontal disease. Therefore, preventative strategies should be employed to reduce the prevalence of the disease.

The results of self-reported symptoms of women enrolled in our study were in agreement with the previous studies on postpartum women. Thomas et al. [14] showed 
Table 2 Logistic regression model of the association between periodontal disease and periodontal symptoms

${ }^{a}$ Periodontal disease was defined as having at least 3 or more sites $\geq 4 \mathrm{~mm}$ CAL

\begin{tabular}{|c|c|c|c|c|}
\hline & \multirow[t]{2}{*}{ Total $(N=409) n(\%)$} & \multicolumn{2}{|c|}{ Periodontal disease $^{\mathrm{a}}$} & \multirow[t]{2}{*}{ Odds ratio $(95 \% \mathrm{CI})$} \\
\hline & & No $(N=158)$ & Yes $(N=251)$ & \\
\hline \multicolumn{5}{|c|}{ Halitosis } \\
\hline No & $251(61.4)$ & $104(41.4)$ & $147(58.6)$ & 1.0 \\
\hline Yes & $158(38.6)$ & $54(34.2)$ & $104(65.8)$ & $1.4(0.9-2.1)$ \\
\hline \multicolumn{5}{|c|}{ Gum bleeding } \\
\hline No & $119(29.1)$ & $49(41.2)$ & $70(58.8)$ & 1.0 \\
\hline Yes & $290(70.9)$ & 109 (37.6) & $181(62.4)$ & $1.2(0.8-1.8)$ \\
\hline \multicolumn{5}{|c|}{ Gum swelling } \\
\hline No & $331(80.9)$ & $135(40.8)$ & $196(59.2)$ & 1.0 \\
\hline Yes & $78(19.1)$ & $23(29.5)$ & $55(70.5)$ & $1.7(0.9-2.8)$ \\
\hline \multicolumn{5}{|c|}{ Tooth mobility } \\
\hline No & $370(90.5)$ & $147(39.7)$ & $223(60.3)$ & 1.0 \\
\hline Yes & $39(9.5)$ & $11(28.2)$ & $28(71.8)$ & $1.7(0.8-3.5)$ \\
\hline
\end{tabular}

Table 3 Logistic regression model of the association between periodontal disease, gum swelling and adverse pregnancy outcome

\begin{tabular}{|c|c|c|c|}
\hline & \multicolumn{2}{|c|}{ Adverse pregnancy outcome ${ }^{a}$} & \multirow{2}{*}{$\begin{array}{l}\text { Odds ratio } \\
(95 \% \mathrm{CI})\end{array}$} \\
\hline & No $(N=285)$ & Yes $(N=124)$ & \\
\hline \multicolumn{4}{|c|}{ Periodontal disease $\mathrm{e}^{\mathrm{b}}$} \\
\hline No & 117 & 41 & 1.0 \\
\hline Yes & 168 & 83 & $0.7(0.5-1.1)$ \\
\hline \multicolumn{4}{|c|}{ Gum swelling } \\
\hline No & 227 & 104 & 1.0 \\
\hline Yes & 58 & 20 & $1.3(0.8-2.3)$ \\
\hline
\end{tabular}

${ }^{a}$ Adverse pregnancy outcome was considered having preterm birth and/or low birth weight

${ }^{\mathrm{b}}$ Periodontal disease was defined as having at least 3 or more sites $\geq 4 \mathrm{~mm} \mathrm{CAL}$

that in a group of 445 postnatal Australian women, 59.5\% reported gum bleeding. Similarly, in a recent study from the UK [8], $44 \%$ of patients $(N=206)$ had gingival bleeding. Of note, self-reported oral hygiene habits were better among Australian and English women when compared with our findings; Australian and English women brushed their teeth at least once a day in 91 and $73.7 \%$ of cases, respectively.

When periodontal disease and adverse pregnancy outcome was considered, no significant associations were observed. This is in accordance with other two Italian studies that evaluated the possible relationship between periodontitis and poor obstetric outcome.

One limitation to note for this study is that data on periodontal symptoms might have been affected by the hormonal changes that occur during pregnancy. As such, self-reported swelling and bleeding could have been related, not only to the presence of periodontal disease, but also to hormonal fluctuations.
Given the association between periodontal disease and self-perceived periodontal disease in postpartum women (OR 1.7), such as gum swelling and tooth mobility, we evaluated previous studies that collected data on periodontal symptoms [8, 14-17]. Interestingly, none of these works asked pregnant women if they had experienced tooth mobility or reported a sensation of swollen gums. These findings allow us to draw several conclusions. First, prenatal medical care providers and dentists may target pregnant women who might be at higher risk for periodontal disease by asking whether they have tooth mobility and/or swollen gums. Second, the dental profession is in an excellent position to play a major role in the prevention of periodontal diseases and educate their patients about the benefits of good oral hygiene practices. Finally, even though we did not find any association, past research has tied poor dental health to increased risk of adverse pregnancy outcome. As such, in the absence of definitive data on the association between periodontitis and adverse pregnancy outcome, clinicians should educate pregnant women about the importance of maintaining a good standard for oral health.

Acknowledgments This study was supported through a grant from the Italian Ministry of University and Research-PRIN 2008: "Clinical and biochemical markers for inflammation and oxidative stress in pregnant women with premature delivery"

Conflict of interest None.

\section{References}

1. Petersen PE (2003) The World Oral Health Report 2003: continuous improvement of oral health in the 21 st century-the approach of the WHO Global Oral Health Programme. Commun Dent Oral Epidemiol 31(Suppl 1):3-23 
2. Laine MA (2002) Effect of pregnancy on periodontal and dental health. Acta Odontol Scand 60(5):257-264

3. Sooriyamoorthy M, Gower DB (1989) Hormonal influences on gingival tissue: relationship to periodontal disease. J Clin Periodontol 16(4):201-208

4. Offenbacher S, Jared HL, O'Reilly PG, Wells SR, Salvi GE, Lawrence HP, Socransky SS, Beck JD (1998) Potential pathogenic mechanisms of periodontitis associated pregnancy complications. Ann Periodontol 3(1):233-250

5. Wan AK, Seow WK, Purdie DM, Bird PS, Walsh LJ, Tudehope DI (2001) Oral colonization of Streptococcus mutans in sixmonth-old predentate infants. J Dent Res 80(12):2060-2065

6. Berkowitz RJ (2006) Mutans streptococci: acquisition and transmission. Pediatr Dent 28(2):106-109 (discussion 192-108)

7. Villa A, Abati S, Strohmenger L, Pileri P, Calabrese S, Cetin I Treatment of periodontal disease and adverse pregnancy outcome: a clinical study (submitted)

8. Hullah E, Turok Y, Nauta M, Yoong W (2008) Self-reported oral hygiene habits, dental attendance and attitudes to dentistry during pregnancy in a sample of immigrant women in North London. Arch Gynecol Obstet 277(5):405-409

9. Zabor EC, Klebanoff M, Yu K, Zhang J, Nansel T, Andrews W, Schwebke J, Jeffcoat M (2010) Association between periodontal disease, bacterial vaginosis, and sexual risk behaviours. J Clin Periodontol 37(10):888-893

10. Pitiphat W, Garcia RI, Douglass CW, Joshipura KJ (2002) Validation of self-reported oral health measures. J Public Health Dent 62(2):122-128
11. Gilbert GH, Litaker MS (2007) Validity of self-reported periodontal status in the Florida dental care study. J Periodontol 78(7 Suppl):1429-1438

12. Eke PI, Dye B (2009) Assessment of self-report measures for predicting population prevalence of periodontitis. J Periodontol 80(9):1371-1379

13. Blicher B, Joshipura K, Eke P (2005) Validation of self-reported periodontal disease: a systematic review. J Dent Res 84(10): $881-890$

14. Thomas NJ, Middleton PF, Crowther CA (2008) Oral and dental health care practices in pregnant women in Australia: a postnatal survey. BMC Pregnancy Childbirth 8:13

15. Wandera MN, Engebretsen IM, Rwenyonyi CM, Tumwine J, Astrom AN (2009) Periodontal status, tooth loss and self-reported periodontal problems effects on oral impacts on daily performances, OIDP, in pregnant women in Uganda: a cross-sectional study. Health Qual Life Outcomes 7:89

16. Christensen LB, Jeppe-Jensen D, Petersen PE (2003) Selfreported gingival conditions and self-care in the oral health of Danish women during pregnancy. J Clin Periodontol 30(11): 949-953

17. Honkala S, Al-Ansari J (2005) Self-reported oral health, oral hygiene habits, and dental attendance of pregnant women in Kuwait. J Clin Periodontol 32(7):809-814 